\title{
Advanced detection strategies for cardiotropic virus infection in a cohort study of heart failure patients
}

\author{
Paul J. Hanson ${ }^{1,2}{ }^{凶}$, Felicia Liu-Fei ${ }^{1}$, Taylor A. Minato ${ }^{1}$, Al Rohet Hossain ${ }^{1}$, Harpreet Rai ${ }^{3}$, Victoria A. Chen ${ }^{1}$, Coco Ng ${ }^{1}$, Kjetil Ask ${ }^{4}$,
} Jeremy A. Hirota ${ }^{4}$ and Bruce M. McManus ${ }^{1,2,5}$

(c) The Author(s), under exclusive licence to United States and Canadian Academy of Pathology 2021

The prevalence and contribution of cardiotropic viruses to various expressions of heart failure are increasing, yet primarily underappreciated and underreported due to variable clinical syndromes, a lack of consensus diagnostic standards and insufficient clinical laboratory tools. In this study, we developed an advanced methodology for identifying viruses across a spectrum of heart failure patients. We designed a custom tissue microarray from 78 patients with conditions commonly associated with virus-related heart failure, conditions where viral contribution is typically uncertain, or conditions for which the etiological agent remains suspect but elusive. Subsequently, we employed advanced, highly sensitive in situ hybridization to probe for common cardiotropic viruses: adenovirus 2, coxsackievirus B3, cytomegalovirus, Epstein-Barr virus, hepatitis C and E, influenza B and parvovirus B19. Viral RNA was detected in $46.4 \%$ (32/69) of heart failure patients, with $50 \%$ of virus-positive samples containing more than one virus. Adenovirus 2 was the most prevalent, detected in $27.5 \%(19 / 69)$ of heart failure patients, while in contrast to previous reports, parvovirus B19 was detected in only $4.3 \%$ (3/69). As anticipated, viruses were detected in $77.8 \%$ (7/9) of patients with viral myocarditis and $37.5 \%(6 / 16)$ with dilated cardiomyopathy. Additionally, viruses were detected in $50 \%$ of patients with coronary artery disease (3/6) and hypertrophic cardiomyopathy (2/4) and in 28.6\% (2/7) of transplant rejection cases. We also report for the first time viral detection within a granulomatous lesion of cardiac sarcoidosis and in giant cell myocarditis, conditions for which etiological agents remain unknown. Our study has revealed a higher than anticipated prevalence of cardiotropic viruses within cardiac muscle tissue in a spectrum of heart failure conditions, including those not previously associated with a viral trigger or exacerbating role. Our work forges a path towards a deeper understanding of viruses in heart failure pathogenesis and opens possibilities for personalized patient therapeutic approaches.

Laboratory Investigation (2022) 102:14-24; https://doi.org/10.1038/s41374-021-00669-4

\section{INTRODUCTION}

Heart failure constitutes one of the more prominent cardiovascular (CV)-related health issue(s) worldwide and in North America, with roughly 6.2 million (2.42\%) Americans over 20 years of age 1 and 669,600 (3.6\%) Canadians over 40 years of age ${ }^{2}$ living with CV disease. These numbers are expected to be sustained in the coming years as CV ailments remain the world's leading causes of morbidity and mortality. Yet, our understanding of the influence of infectious agents as initial triggers and in exacerbation of morbidity and mortality remains meagre.

The contribution of cardiotropic viruses in acute and chronic manifestations of heart failure have become increasingly apparent, although they are likely significantly underreported ${ }^{3-6}$. The role of classically studied cardiotropic viruses such as enteroviruses has been known for considerable time ${ }^{7}$; however, even their contribution to acute and chronic heart failure is likely underrepresented and unconfirmed, given that the standard of practice in diagnostics is quite insensitive, non-specific, and typically do not identify the causative infectious agent ${ }^{8,9}$. Moreover, viruses that were once considered primarily to cause respiratory illness, such as influenza ${ }^{10}$ and coronaviruses ${ }^{11,12}$, are becoming increasingly appreciated in the context of virus-induced heart failure. Many of these viruses are also associated with outbreaks of pandemic scale ${ }^{13-16}$, raising the level of urgency to enhance our knowledge base of viral prevalence and contribution to acute and chronic heart failure. Indeed, many clinical manifestations of heart failure may be attributable to virus infection as an initial trigger, which is especially apparent in those who exhibit associated CV diseases such as myocarditis and dilated cardiomyopathy (DCM) ${ }^{3,4}$.

Myocarditis, which can present as acute heart failure among other signs and symptoms, is commonly caused by infection with an array of viruses including: enteroviruses ${ }^{7}$, adenoviruses ${ }^{17}$, parvovirus B19 $(\mathrm{B} 19 \mathrm{~V})^{18}$, hepatitis $\mathrm{C}(\mathrm{HCV})^{19}$ and $\mathrm{E}(\mathrm{HEV})^{20}$, Epstein-Barr virus $(E B V)^{21}$, influenza viruses $(A \text { and } B)^{10,22-24}$, and human immunodeficiency virus (HIV) ${ }^{3,5}$. Cardiotropic viruses have also been demonstrated as an initial trigger which may to lead to and exacerbate chronic conditions. For example, enteroviruses have been detected in up to $40 \%$ of patients diagnosed with $\mathrm{DCM}^{4}$. Another study detected B19V in endomyocardial biopsies

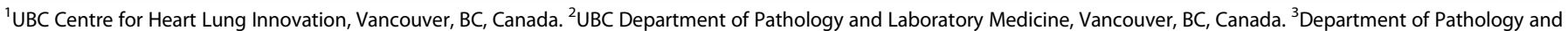

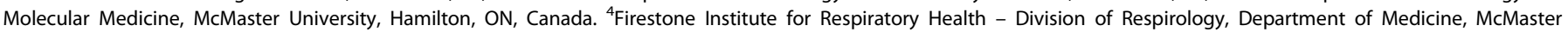
University, Hamilton, ON, Canada. ${ }^{5}$ PROOF Centre of Excellence, Vancouver, BC, Canada. ${ }^{凶}$ email: paul.hanson@hli.ubc.ca

Received: 6 May 2021 Accepted: 20 August 2021

Published online: 4 October 2021 
of $51.4 \%$ of patients with idiopathic DCM via polymerase chain reaction (PCR) and found that the virus was often associated with concurrent co-infection ${ }^{25}$. In addition, myocarditis has frequently been described in patients with arrhythmogenic right ventricular cardiomyopathy (ARVC), primarily considered a genetic disorder characterized by fibrofatty replacement of the myocardium. ARVC has been reported as a common form of exercise-related heart failure in young adults. It has been postulated that recurring bouts of myocarditis observed in ARVC are attributable to a viral trigger in acquired forms of ARVC. Indeed, several studies have reported the detection of cytomegalovirus (CMV), HCV and B19V in sporadic ARVC, implicating cardiotropic viruses in these patients ${ }^{26,27}$. Others have suggested that genetic forms of ARVC only present phenotypically in the presence of a viral trigger ${ }^{26}$.

Globally, it is estimated that more than 70 million people have been infected with HCV, which is becoming increasingly associated with subclinical and clinical manifestations of CV disease ${ }^{28,29}$. Likewise, influenza viruses are becoming progressively linked to heart failure and patient prognosis. In 2013-14, of 218,540 patients hospitalized for influenza, 45,460 (20.8\%) demonstrated accompanying heart failure; these patients also had higher in-hospital mortality ${ }^{30}$. Further, a surveillance study of 451,588 hospitalized adults found a $5 \%$ increase in influenza activity to be associated with a $24 \%$ increase in heart failure hospitalization rates ${ }^{31}$, suggesting that heart failure and complications thereof are attributable to influenza infections.

With the emergence of SARS-CoV-2 and resultant COVID-19 pandemic, recent research has turned to studying the effects of the novel coronavirus on the CV system. Heart failure has been noted as a common complication of COVID-19 $9^{32,33}$, considering its presence in $49 \%$ of deceased patients ${ }^{34}$. Studies of past coronavirus and influenza epidemics suggest that viral infections can lead to exacerbation of heart failure by triggering a significant inflammatory response and localized vascular inflammation ${ }^{35}$. However, considering the novelty of COVID-19, there remains much room to study the mechanisms by which the new coronavirus may cause heart failure.

In addition to well-established conditions such as myocarditis and DCM, for which viral etiology has been established, inflammatory conditions such cardiac sarcoidosis ${ }^{36}$ and giant cell myocarditis $(\mathrm{GCM})^{16}$ represent pathological enigmas for which the underlying etiology is unknown. Such has made therapeutic options largely supportive and exceedingly limited ${ }^{37}$. It has been postulated that both sarcoidosis and $\mathrm{GCM}^{38}$ may originate from infectious agents ${ }^{39}$, yet to date this theory has not been bolstered by evidence. Additionally, systemic viral infections may involve the heart. HCV in particular has been linked to a spectrum of extrahepatic manifestations, including myocarditis, cardiomyopathies and coronary artery disease ${ }^{40}$. Indeed, a higher prevalence of HCV infection has been observed among patients with hypertrophic cardiomyopathy $(\mathrm{HCM})^{41}$, a condition largely defined as a genetic disease. This latter evidence further suggests a role for viral infections across a broad spectrum of heart failure manifestations.

Yet, our understanding and interpretation of data linking viral presence to causation of heart failure is incomplete. Viral infection is clearly correlated to cases of heart failure, but direct evidence indicating viruses play a significant role as etiologic agents in the development and progression of heart failure is insufficient.

At the present, a definitive tissue-based diagnosis (i.e., confirmation of virus in the heart) is not standard practice to diagnose acute virus-related heart failure such as myocarditis ${ }^{42}$. As well, viral presence in the heart is rarely confirmed in chronic conditions such as DCM, with a default diagnostic designation usually being "idiopathic" ${ }^{\prime 3}$. If suspected, a diagnosis is commonly made based upon clinical suspicion of infection (i.e., recent viral prodrome and flu-like illness), elevated non-specific markers of cardiac injury (i.e., blood troponin levels) and antibodies against a given virus ${ }^{42,44}$. To confirm viral presence, $P C R$ is often the standard used to detect viral genome in the heart ${ }^{45}$. However, PCR is insufficient to detect viral RNA in the heart in a manner that would affirm causation, as there is the potential for false positivity ${ }^{46}$ due to viral presence in the blood being indistinguishable from viral presence in tissue using this method ${ }^{47}$. The pervasiveness of more common viruses further complicates matters. For example, EBV is among the most prevalent and ubiquitous infections worldwide, with an estimated $95 \%$ of the population infected ${ }^{48}$. As such, it is difficult to determine whether EBV infection is causative under certain conditions of heart failure or whether it is merely a bystander. Although EBV has been implemented in rare cases of acute myocarditis ${ }^{49}$, exacerbation of coronary events ${ }^{50}$ and heart failure ${ }^{51}$, definitive evidence of causation remains elusive and such studies have relied on detection of neutralizing antibodies for EBV in patient blood. Similar analyses have been conducted for B19V, with limited evidence for causation or contribution to acute and chronic cardiac disease $^{47,52}$.

In efforts to address the foregoing gaps in understanding noted above, we developed a tissue microarray (TMA) from a cohort of heart failure patients at St. Paul's Hospital in Vancouver, BC, with common inflammatory (viral myocarditis, lymphocytic myocarditis, eosinophilic myocarditis, sarcoidosis, giant cell myocarditis $(\mathrm{GCM})$ ) and non-inflammatory (HCM, DCM, ARVC and coronary artery disease (CAD)) conditions. With the TMA, we performed high-throughput analyses for a panel of viruses commonly implicated in acute and chronic heart failure (coxsackievirus B3 (CVB3), B19V, influenza B (Inf B), CMV, HCV, HEV, adenovirus 2 (AAV2) and EBV). We applied RNAscope ${ }^{\circledR}$, in situ hybridization techniques to detect actively replicating viruses. The method of interrogation is uniquely capable of detecting low copy number RNA. This approach corrects for and addresses the challenge of proving causation by providing spatial context, revealing where in the tissue the viral nucleic acids are present. Indeed, the advanced technique used in this study make possible the detection of a single copy of RNA. By screening for and identifying viruses in a variety of cardiac diseases, we aim to shed new light on the contribution of viruses in the setting of acute and chronic heart muscle diseases.

\section{METHODS}

\section{Tissue microarray design and development}

TMA design. A TMA (TMA Master II, 3D Histech, Budapest Hungary) consisting of representative tissues from common forms of heart failure was designed and developed utilizing clinically annotated archival tissues available from the Cardiovascular Tissue Registry (CVTR) (Center for Heart Lung Innovation, St. Paul's Hospital at the University of British Columbia) under ethical protocols (H05-50004, H15-40080) approved by the University of British Columbia's Clinical Research Ethics Board. All specimens were deidentified before use in the study, and the patients or appropriate parties provided written, informed consent when given the option to contribute to clinical research. The TMA design included specimens from 78 different patients with varying diagnoses, including 6 with CAD, 22 with DCM (16 idiopathic, 6 with chronic active myocarditis (CAM)), 4 with ARVC, 4 with HCM, 7 with transplant rejection (TR), 4 with sarcoidosis, 5 with eosinophilic myocarditis, 9 with confirmed viral myocarditis, 6 with lymphocytic myocarditis of uncertain etiology, 2 with GCM and 9 healthy controls (Fig. 1).

Diagnostic criteria. Patients with archival tissues available from the CVTR and associated clinical pathology reports including pathologist-confirmed diagnoses of interest were appraised. Specimens were identified by searching the biobank's digital patient filing system. Multiple synonymous search terms were used for each diagnosis in order to identify all matching specimens. Patient age at tissue donation and sex were obtained from pathology reports where available. Exclusion criteria included any pathology report and specimen with ambiguous or unclear diagnoses and those with multiple primary diagnoses, which limited sample size. 
A
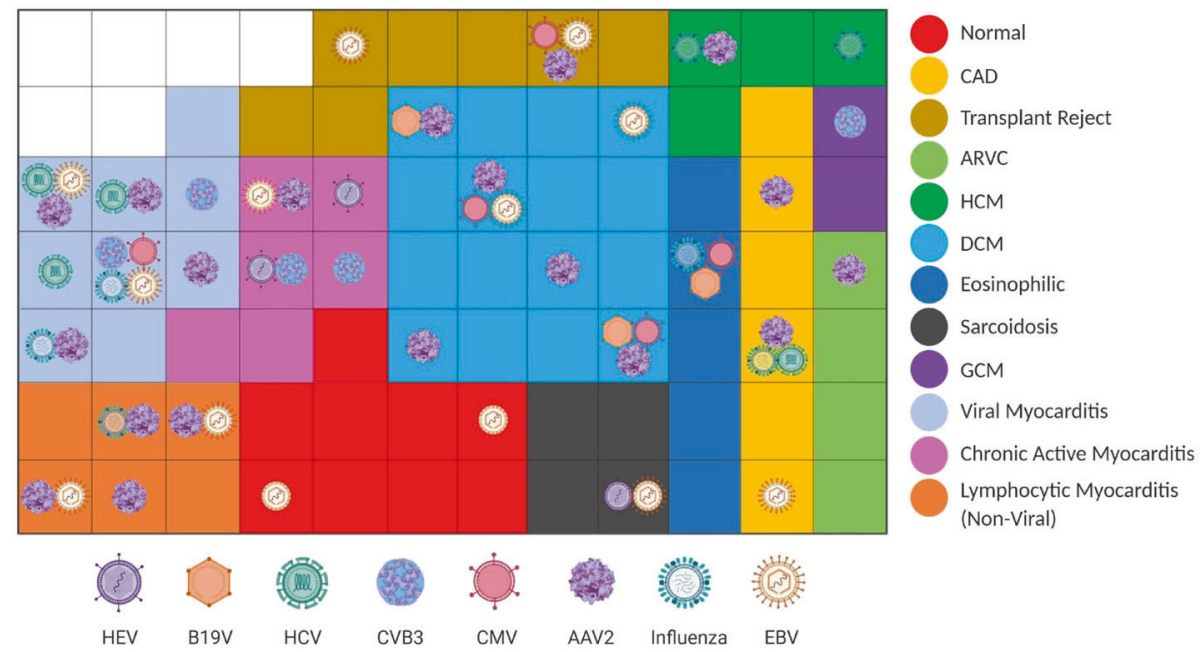

B
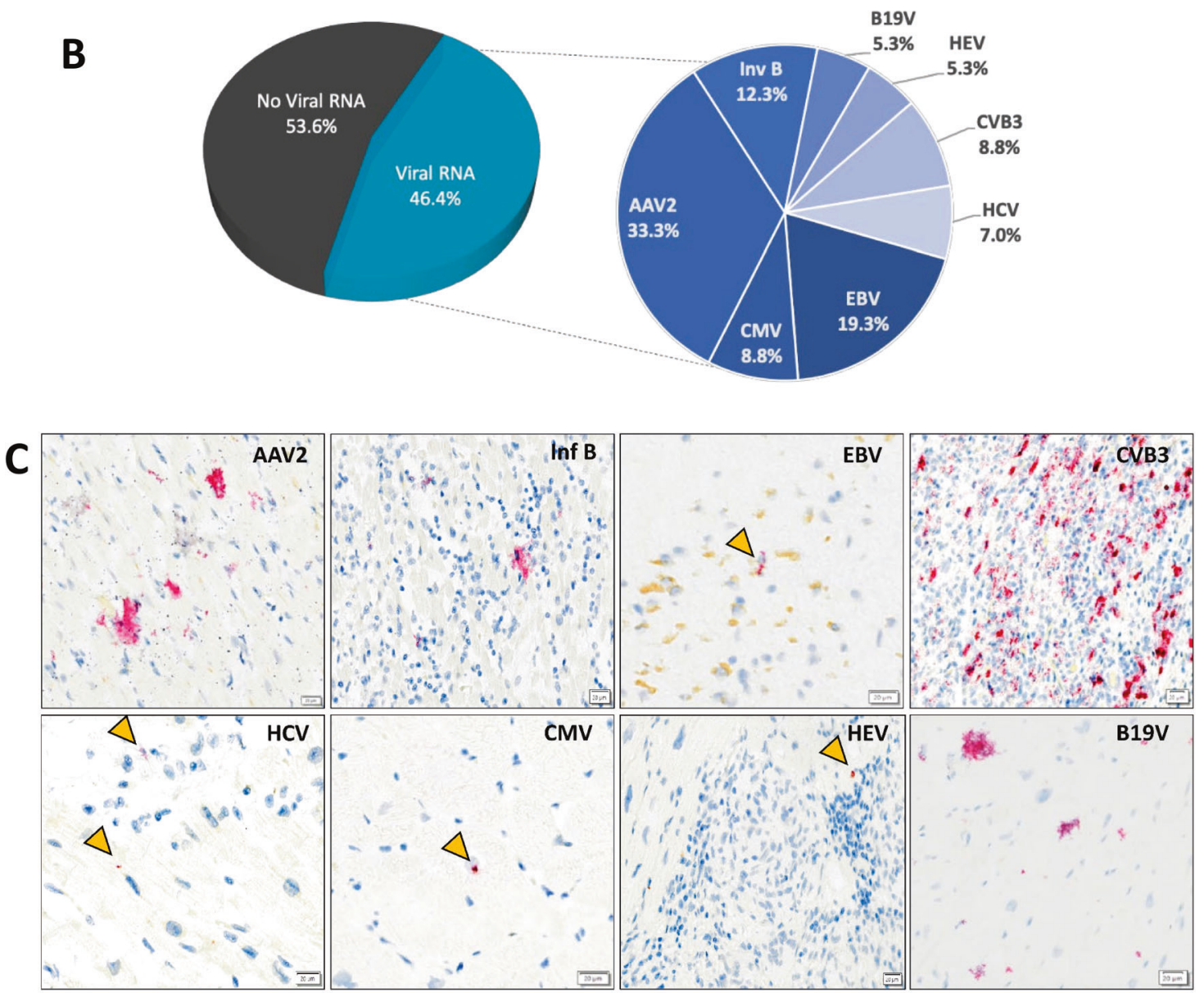

Fig. 1 Detection of cardiotropic viruses in a human heart failure cohort via RNAscope . A Schematic diagram of TMA representing the individual patient diagnoses with the associated viruses present. Each block represents individual patients with their respective diagnoses and detected viruses. CAD indicates coronary artery disease, TR indicates transplant rejection, ARVC arrhythmogenic right ventricular cardiomyopathy, HCM hypertrophic cardiomyopathy, DCM dilated cardiomyopathy, and GCM giant cell myopathy. B Proportion of viral infections in a cohort of 69 heart failure patients and the distribution of different viruses detected via RNAscope . C Representative positive tissues (red signal) for the corresponding virus via in situ hybridization are as follows: Human Adenovirus 2 (AAV2) in clinically diagnosed viral myocarditis, Hepatitis C virus (HCV) in clinically diagnosed viral myocarditis, Influenza B (Inf B) in clinically diagnosed viral myocarditis, Cytomegalovirus (CMV) in clinically diagnosed dilated cardiomyopathy, Epstein-Barr virus (EBV) in clinically diagnosed coronary artery disease (red) with lipofuscin accumulation seen in the surrounding yellow/brown pigment, Hepatitis $E$ virus (HEV) in clinically diagnosed chronic active myocarditis, coxsackievirus B3 (CVB3) in clinically diagnosed viral myocarditis, Parvovirus B19 (B19V) in clinically diagnosed dilated cardiomyopathy. Yellow arrows indicate regions of viral RNA, scale bars are $20 \mu \mathrm{m}$. 
Specimens with a desired primary diagnosis on the background of other chronic or remote secondary CV diseases were included. The heterogeneity of the patient cohort is reflective of the spectrum of and distribution of diagnoses for heart failure patients at our Center, with the a diagnosis of DCM accounting for the greatest proportion of patients.

Tissue sections. Deidentified tissues from the left ventricular free wall of explanted hearts, ventricle assist device core tissue, and autopsy specimens from patients meeting the diagnostic criteria (above) were identified within the CVTR biobank repository. All specimens were stored as formalin-fixed paraffin-embedded (FFPE) blocks - tissues were fixed in formalin for at least 24 and up to $48 \mathrm{~h}$ prior to being embedded in paraffin blocks. Definitive diagnoses were made before transplant or at the time of autopsy. Hearts from transplant recipients were transferred into fixative on average within $1 \mathrm{~h}$ of explantation and autopsy specimens were transferred into fixative typically between 4 and $24 \mathrm{~h}$ post mortem. FFPE blocks were chosen as the appropriate method of preservation due to their ability to retain tissue morphology. Any loss in antigenicity, essential for the detection of protein markers, was counteracted by antigen retrieval techniques before performing immunohistochemical (IHC) staining.

Cardiovascular pathologist review. A $4 \mu \mathrm{m}$ FFPE section from each of the specimens was placed onto a slide, deparaffinized and rehydrated. Tissues were stained with hematoxylin and eosin and reviewed by a CV pathologist (Dr. McManus) blinded to diagnosis to identify regions of pathological significance. Selected regions corresponding to the histological hallmarks of a given diagnosis were identified and photographed, and the region on the original FFPE block was noted.

Tissue coring and TMA development. The $2 \mathrm{~mm}$ regions of pathological significance (above) or sections representative of normal, healthy tissue (controls) from all 78 patients were cored from the original FFPE blocks and transferred to the recipient block to generate a custom TMA employed in the experimental work that follows. The tissue specimens included at least 3 biological replicates $(n \geq 3)$ for all diagnoses except $\operatorname{GCM}(n=2)$. The TMA design and layout, and the designation of each tissue by diagnosis are depicted in the sector map in Fig. $1 \mathrm{~A}$.

\section{Detection of cardiotropic viruses via RNAscope ${ }^{\circledR}$ in situ hybridization}

Four-micron sections from the TMA (described above) were placed onto slides, deparaffinized in xylene and rehydrated. Slides were probed via singleplex RNAscope 2.5 LSX RED kit (cat. \#322750) for the viruses of interest: CVB3, B19V, Inf B, CMV, HCV, HEV, AAV2 and EBV (Table 1). Slides were counterstained with hematoxylin, then digitally scanned using Aperio $^{\mathrm{TM}}$ ImageScope ${ }^{\mathrm{TM}}$. Representative images were captured for each diagnosis and corresponding virus. Viral positivity was assessed and documented for each core (Fig. 1C).

\section{RESULTS}

Cardiotropic viruses detected across a spectrum of conditions in a heart failure cohort via RNAscope ${ }^{\circledR}$

Within our cohort, cardiotropic viral RNA was detected in $43.6 \%$ (34/78) of patient specimens (including 2/9 normal controls) via RNAscope ${ }^{\circledR}$, indicating active infection (Fig. 1). Among patients with clinically diagnosed heart failure, viral RNA was detected in $46.4 \%(32 / 69)$ of patients. Out of 78 total tissues analyzed (including $n=9$ normal), virus-positive detections included: EBV $(n=13), \mathrm{CMV}(n=5), \mathrm{AAV} 2(n=19)$, Inf B $(n=7), \mathrm{B} 19 \mathrm{~V}(n=3), \mathrm{HEV}$ $(n=3)$, CVB3 $(n=5)$ and HCV $(n=4)$. Of note, most diagnostic categories showed between $20-78 \%$ virus positivity, including the detection of EBV in $22.2 \%$ (2/9) of normal, healthy control heart tissue. $21.8 \%(17 / 79)$ of all specimens, or $50 \%(17 / 34)$ of all viruspositive specimens, tested positive for more than one virus. AAV2 was the most prevalent virus and was detected in $27.5 \%(19 / 69)$ of all heart failure patients irrespective of diagnosis (Fig. 1). Of the 32 heart failure patients with replicative strands of viral RNA detected in their tissues, $53.1 \%(n=17)$ tested positive for two or more viruses, which was most commonly associated with AAV2 infection. AAV2 was prevalent among patients clinically diagnosed with lymphocytic myocarditis of "non-viral" origin, being detected in $66.7 \%$ of patients (4/6). Additionally, AAV2 was detected in $44.4 \%$ (4/9) of patients clinically diagnosed with viral myocarditis (based on exhibition of clinical symptoms associated with viral infection), with $75 \%$ (3/4) of AAV2-positive patients having multiple, concurrent viral infections detected in addition to AAV2. AAV2 was detected in $16.7 \%(1 / 6)$ of patients with chronic active myocarditis, which was also detected in conjunction with EBV. Moreover, AAV2 was detected in $31.3 \%(5 / 16)$ of patients with clinically diagnosed DCM. AAV2 was also detected in 25\% (1/4) of patients with HCM, 25\% (1/4) of patients diagnosed with ARVC and $14.3 \%(1 / 7)$ of patients with cardiac transplant rejection (Table 2).

\section{A range of viruses were detected in patients with clinically diagnosed acute viral myocarditis}

In patients with a primary diagnosis of acute or fulminant viral myocarditis at transplant or autopsy, viral RNA was detected in $77.8 \%(7 / 9)$ of patients. The average patient age was 37 years old and the majority $(75.0 \%, 6 / 8)$ were female (Table 1$)$. With our panel of viruses, viral RNA was not detected in 2 of 9 patients with clinically diagnosed viral myocarditis. CVB3, typically considered among the most common enteroviruses associated with myocarditis $^{53-55}$, was detected in $22.2 \%$ (2/9) of confirmed viral myocarditis specimens in our cohort. AAV2 was the most commonly detected virus among patients in this group at $44.4 \%$ (4/9) and was associated with concurrent infection in 3 of 4 positive patients (Fig. 2). HCV was also detected in $33.3 \%$ (3/9) of patients, with AAV2 detected concurrently in 2 of 3 patients with HCV infection. EBV was detected in $22.2 \%$ (2/9) of viral myocarditis patients and in each instance was associated with concurrent infection (one patient with HCV, AAV2 and EBV, and another patient with CVB3, CMV, Inf $B$ and EBV) (Figs. 1 and 2).

\section{Viral presence in inflammatory and non-inflammatory dilated cardiomyopathy}

As DCM has traditionally been linked to an initial viral trigger as well as persistent infection and chronic inflammation, DCM patients represented the largest $(n=22)$ group surveyed in our cohort. Patients were subdivided into DCM or DCM with chronic active myocarditis, listed as chronic active myocarditis (CAM). Of the patients with chronic active myocarditis in the context of DCM (CAM), 66.7\% (4/6) were virus-positive, with the following viruses present in tissues: CVB3 33\% (2/6), HEV 33\% (2/6), (including one patient with concurrent CVB3 and HCV infection), and one patient with concurrent AAV2 and EBV co-infection, representing $16.7 \%$ $(1 / 6)$. The inflammatory infiltrate was composed predominantly of T-cells (CD3) with a mixed infiltration of CD4- and CD8-positive cells (Fig. 3). In patients with DCM without active inflammation (DCM), viruses were detected in $37.5 \%(6 / 16)$ of patients. As aforementioned, AAV2 was the most prevalent virus within our cohort of patients with non-inflammatory DCM, with $31.3 \%(5 / 16)$ demonstrating positive viral RNA genomes in their tissue. B19V was detected in $12.5 \%(2 / 16)$ of patients and in both instances was associated with concurrent viral co-infections (AAV2 and CMV). CMV was detected in $12.5 \%$ (2/16) of DCM patients, one of which had multiple viral infections (B19V, CMV and AAV2). Overall, viruses were detected in $45.5 \%(10 / 22)$ of patients with clinically diagnosed DCM (Fig. 1).

\section{Detection of HEV and EBV in a cardiac sarcoidosis lesion}

Among the four patients in our cohort with a primary clinical diagnosis of cardiac sarcoidosis, viral RNA was detected in one patient (25\%). HEV viral RNA was detected in multifocal regions surrounding an inflammatory granulomatous lesion (Fig. 4A), the hallmark of sarcoidosis, in the explanted heart of a 59-year-old male heart transplant recipient. In the same patient, active EBV infection was also detected within the granulomatous lesion 


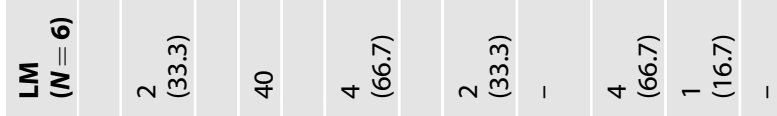

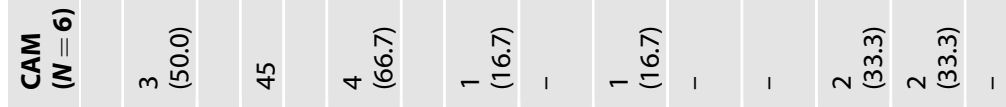

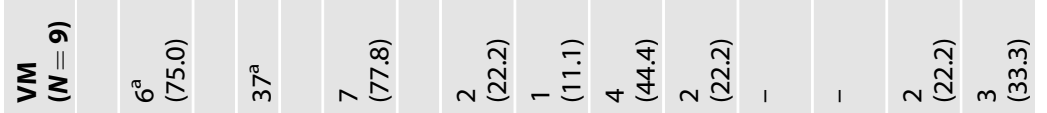

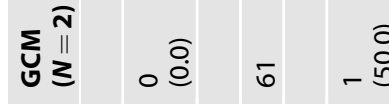

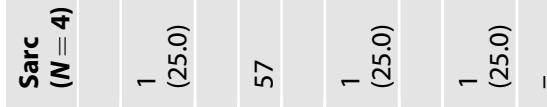

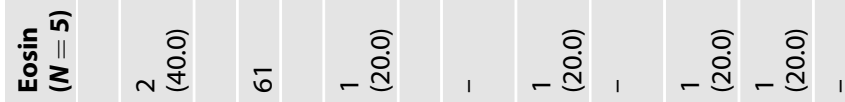

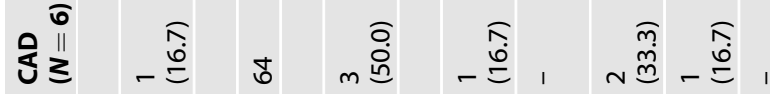

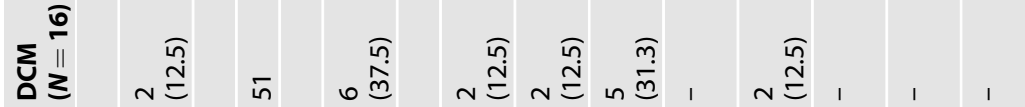

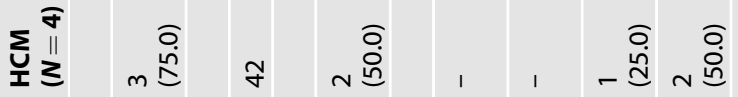

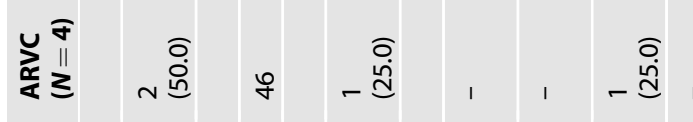

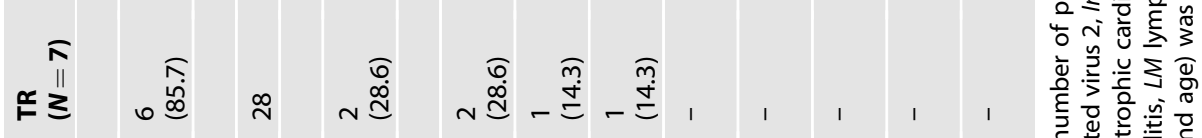

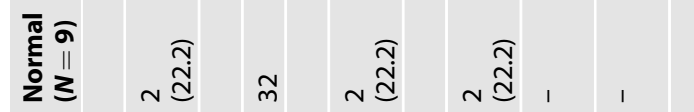

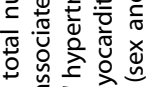
ه

谜交突

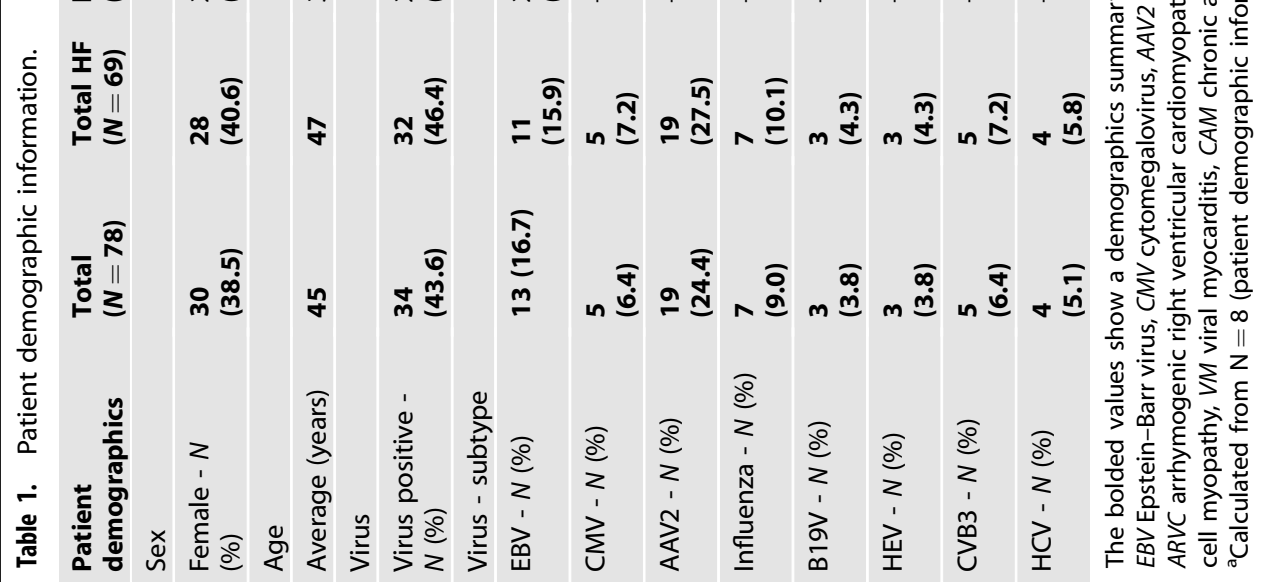


(Fig. 4B). To our knowledge, this is the first description of actively replicating virus within a granulomatous lesion in a patient with cardiac sarcoidosis.

\section{Detection of viral genomes in transplant rejection with spontaneous cardiac sarcoidosis}

Among patients diagnosed with cardiac transplant rejection, viral genomes were detected in $28.6 \%$ (2/7) of patients. Remarkably, EBV, CMV and AAV2 viral genomes were detected in a 38-year-old female patient with spontaneous cardiac sarcoidosis in conjunction with cardiac allograft vasculopathy (Fig. 5) at the time of transplant. EBV was detected in the second specimen with acute transplant rejection at autopsy. This patient was a 14 -year-old female heart transplant recipient, negative for myocarditis.

\section{Detection of coxsackievirus B3 in giant cell myocarditis}

Viral RNA was also detected in a patient with GCM. Of the two patients in our cohort diagnosed with GCM, CVB3 was detected in the explanted heart of a 61-year-old male. Again, to our knowledge, this is the first report of actively replicating virus within the myocardium of a GCM patient.

Table 2. List of in situ hybridization (RNAscope ${ }^{\circ}$ ) Probes.

\begin{tabular}{|lll|}
\hline Virus type & $\begin{array}{l}\text { RNAscope } \\
\text { target region }\end{array}$ & Probe \\
\hline $\begin{array}{l}\text { Adeno-Associated Virus } \\
2 \text { (AAV2) }\end{array}$ & $294-1147$ & 300040 \\
\hline Coxsackievirus B3 (CVB3) & $4-7405$ & 409298 \\
\hline Human Parvovirus B19 (B19V) & $389-2254$ & 434028 \\
\hline Hepatitis C Virus (HCV) & N/A & 318968 \\
\hline Hepatitis E Virus (HEV) & $19-7257$ & 468118 \\
\hline Influenza B (Inf B) & $2-201$ & 313248 \\
\hline Cytomegalovirus (CMV) & $241-1507$ & 300040 \\
\hline Epstein-Barr Virus (EBV) EBNA & $56-1006$ & 485388 \\
\hline Human Positive control & N/A & 322750 \\
\hline Negative DapB control & N/A & 320878 \\
\hline
\end{tabular}
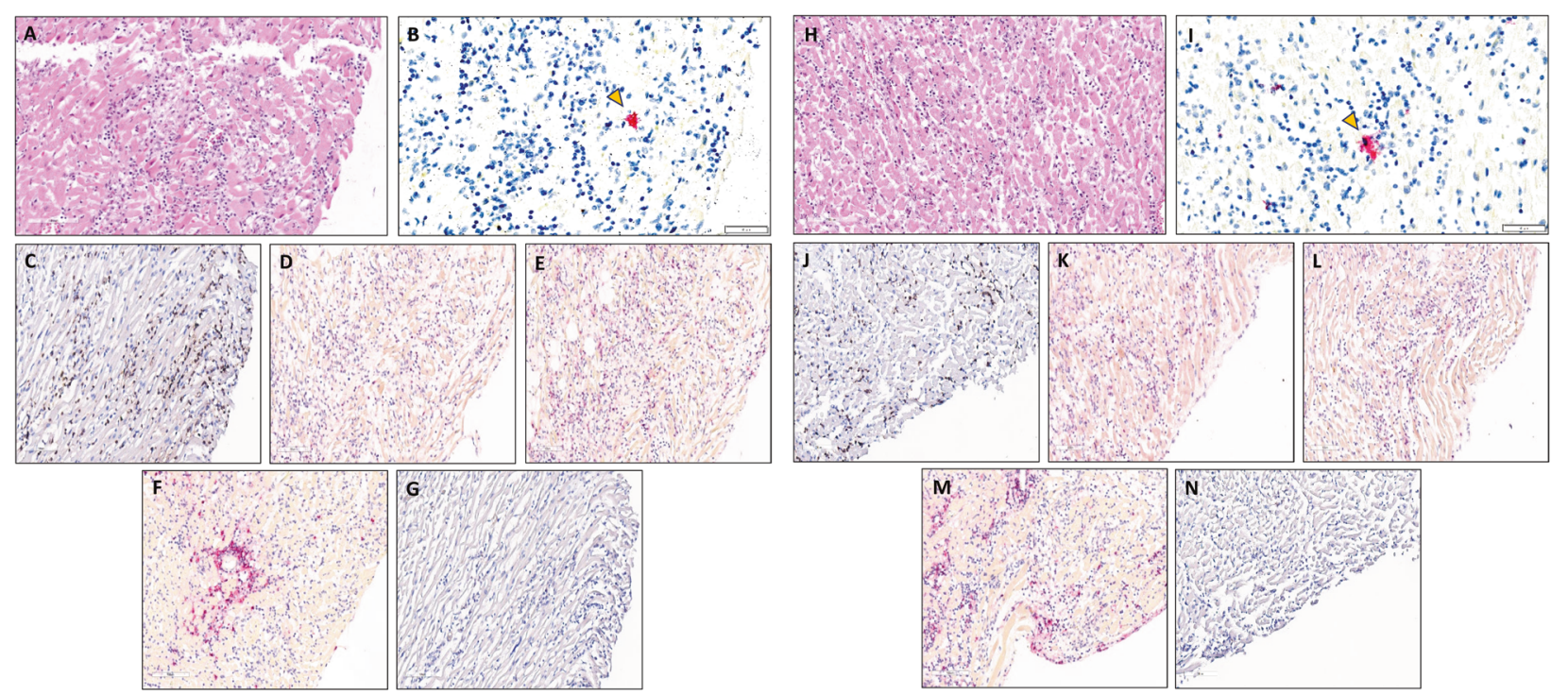

\section{DISCUSSION}

The prevalence and contribution of viruses in the induction and exacerbation of the many forms of heart failure, from acute viral myocarditis and transplant rejection to chronic conditions such as DCM and ischemic, is complex, poorly understood and likely unappreciated. To date, no meta-analyses or even sufficient epidemiological data exists on the presence of viruses across a spectrum of conditions which result in heart failure, and available information is focused on viral influence on specific CV diseases. While the true prevalence of virus-associated heart failure is unknown, the role of viruses and their contribution to heart failure is likely grossly underestimated. Here we report the detection of cardiotropic viral genomes in a cohort of heart failure patients at

Fig. 2 Adeno-associated virus 2 and influenza B in patient with viral myocarditis. A, $\mathbf{H}$ Hematoxylin and Eosin staining of regions with detectable viral RNA in the left ventricular tissue of an explanted heart from patient with clinically confirmed viral myocarditis. B, I Detection of AAV2 and Inf B RNA (red signals) via RNAscope indicated by arrows. IHC staining of regions with detectable viral RNA for CD3 (C, J), CD4 (D, $\mathbf{K})$, CD8 (E, L), CD20 (F, M) and CD68 (G, N). Positive signals are indicated in brown $(\mathbf{C}, \mathbf{F}, \mathbf{G}, \mathbf{J}, \mathbf{M}, \mathbf{N})$ and red $(\mathbf{D}, \mathbf{E}, \mathbf{K}, \mathbf{L})$. Representative images $(\mathbf{C}-\mathbf{G})$ and $(\mathbf{J}-\mathbf{N})$ were captured from regions with detectable AAV2 and Inf B RNA, respectively at 20x magnification. 

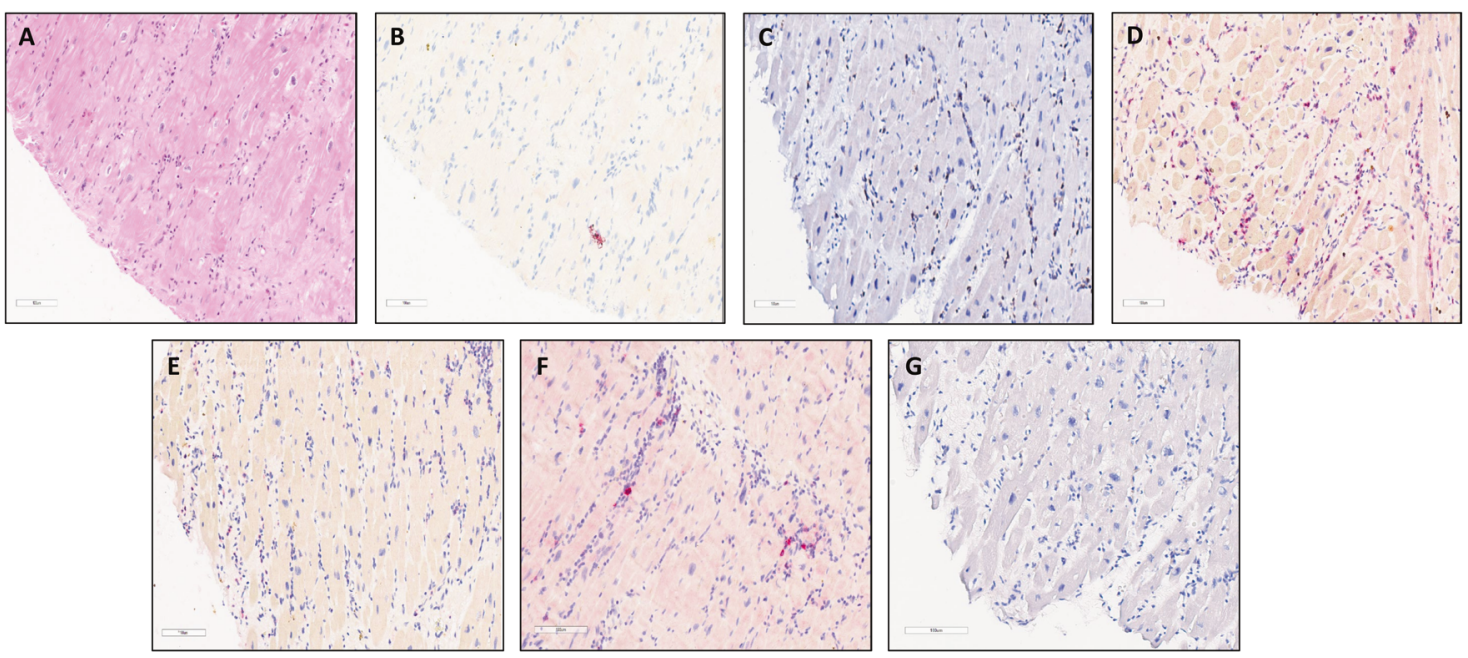

Fig. 3 Hepatitis E Viral (HEV) RNA detected in patient with chronic active myocarditis. A Hematoxylin and Eosin staining of tissue from an explanted heart of a patient with chronic active myocarditis. B Detection of HEV RNA (red signals) via RNAscope indicated by arrows. IHC staining of regions with detectable viral RNA for CD3 (C), CD4 (D), CD8 (E) and CD20 (F) and CD68 (G) as markers of T cell co-receptor, T helper cells, Natural Killer (NK) cells, B cells and macrophages respectively. Positive signals are indicated in brown (C, G) and red (D-F). Representative images (C-G) were captured from regions with detectable HEV RNA at a 20x magnification.

transplant, explant or autopsy with conditions spanning from acute inflammatory myocarditis to primarily genetic conditions such as HCM. The detection of multiple cardiotropic viruses across a spectrum of conditions demonstrates a high rate of positivity currently not appreciated in literature or clinically. Such is reflective of the insufficiency of current diagnostic techniques, highlighting the need for highly sensitive, advanced methods as adjuvant diagnostic testing to identify patients whom viruses may have acted as an initial trigger for or contributed to the exacerbation of heart failure. Using a highly sensitive and specific method of in situ hybridization, we detected viral genome in $46.4 \%$ of heart failure patients in our cohort, regardless of diagnosis (Fig. 1B). These findings highlight the prevalence of viral genomes in the hearts of patients with previously described conditions (i.e., viral myocarditis, chronic active myocarditis, DCM, etc.) as well as heart tissue from conditions for which viruses have not been reported to date (i.e., GCM, sarcoidosis).

\section{A range of viruses detected in viral myocarditis}

Viral myocarditis is perhaps the most well-studied disease entity in the context of virus-associated heart failure, accounting for many sudden and unexpected cardiac deaths in children and young adults and resulting in DCM in its chronic sequalae. Previous studies have demonstrated the detection of viral genomes in $40 \%$ of patients under the age of 18 with myocarditis or $\mathrm{DCM}^{17}$. The types of viruses detected in patients varies geographically, with enteroviruses and adenoviruses being predominantly detected in North America ${ }^{17}, \mathrm{~B} 19 \mathrm{~V}$ being detected at a high rate in Germany ${ }^{56}$, and HCV being most prevalent among those with virus-associated heart failure as reported by Japan ${ }^{57}$. HCV antibodies were detected in $4.4 \%$ of patients in a myocarditis treatment trial, while HCV infection is observed in $1.8 \%$ of the US population, with a variance of $0-15 \%$ regionally ${ }^{19}$. Moreover, globally it is estimated that more than 70 million people have been infected with HCV, which is increasingly being associated with CV disease ${ }^{28}$ and is considered a major cause of viral myocarditis in Japan ${ }^{57}$. Yet still, our epidemiological data is likely vastly under-representative of the true prevalence and contribution of viruses in heart failure, given that the majority of viral infections are asymptomatic or subclinical and are frequently not suspected or recognized as contributing to or exacerbating disease in the context of heart failure.
Confounding these issues, due to our inability to suspect viral involvement from the onset our ability to screen for viruses and diagnose these conditions is severely limited ${ }^{8,9,58}$. Treatment options thus remain mostly supportive, often consisting of mechanical circulatory support and heart transplantation as a means of ensuring patient survival ${ }^{59,60}$.

\section{Virus-mediated exacerbation of cardiac transplant rejection}

Previous studies have implicated the detection of cardiotropic viral genomes, including adenoviruses, B19V, EBV, CMV, enteroviruses and herpes simplex viruses, with adverse outcomes and premature cardiac transplant rejection ${ }^{61,62}$. One study demonstrated that the patients in which viral genomes were detected were 6.5 times more likely to reject the transplanted heart than patients who were virus-negative ${ }^{61}$. Moreover, adenovirus detection was associated with the highest mortality rate among heart transplant recipients who were virus postive ${ }^{61}$. CMV seropositivity was also identified as a risk factor for poor outcomes in the context of transplant rejection ${ }^{63}$. The prevalence and types of viruses identified in the context of transplant rejection has also shifted over time, with enteroviruses giving way to adenoviruses in the 1990s, followed by an increase of B19V in the $2010 \mathrm{~s}^{62}$. In our cohort, we report the detection of cardiotropic viruses in $28.6 \%$ (2/ 7) of patients with cardiac transplant rejection. In both patients, EBV was detected. In one patient, the presence of EBV, CMV and AAV2 were detected (Fig. 5). Interestingly, the patient with concurrent infection by EBV, CMV and AAV2 developed spontaneous, previously unreported cardiac sarcoidosis, which supports the notion of a viral trigger and/or viral involvement in the pathogenesis of cardiac sarcoidosis ${ }^{39}$. Our results merely indicate that the prevalence of viruses in transplant patients is higher than perhaps previously anticipated. However, given the findings in previous studies that viral genome presence results in poor outcomes of graft rejection, the case can be made that standard of care for individual cardiac transplant recipients could include the screening for cardiotropic viruses as a part of their routine endomyocardial biopsy process if causation is further established with future research. Moreover, further consideration and research may be devoted to the treatment of such conditions. However, a delicate balance must be found with patients receiving immunosuppressive therapies. 


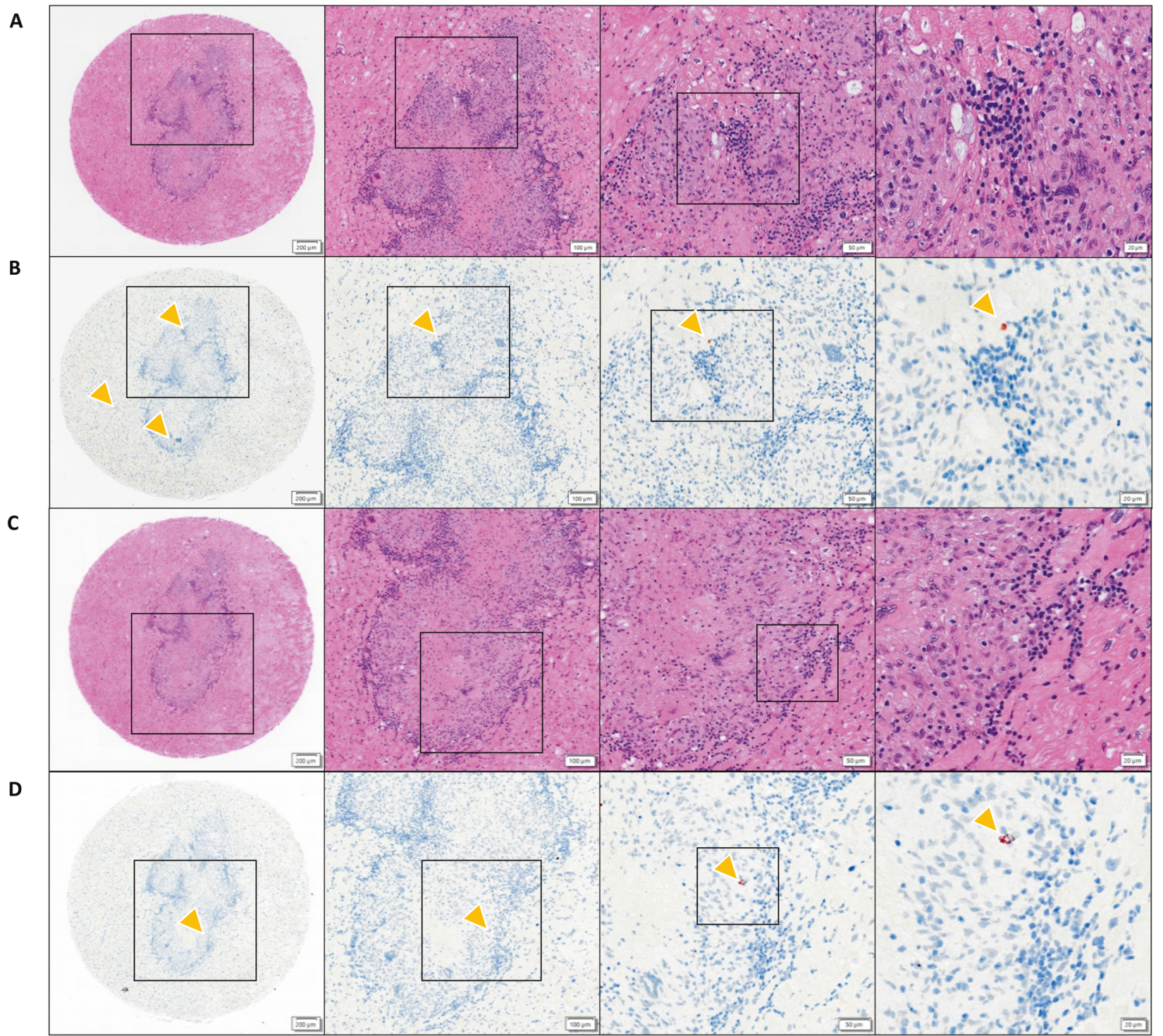

Fig. 4 Hepatitis E and Epstein-Barr viral RNA within granulomatous tissue of cardiac sarcoidosis. A, C Hematoxylin and Eosin staining of a granulomatous lesion within the left ventricle of an explanted heart from patient with cardiac sarcoidosis. B RNAscope ${ }^{\circledR}$ detection of HEV and EBV (D) (red) with a granulomatous lesion, the histological hallmark of cardiac sarcoidosis. The Yellow arrows indicate regions of positive HEV or EBV virus. Images are shown from low to high magnification of the corresponding region on H\&E and RNAscope ${ }^{\circledR}$ scope probe with hematoxylin staining.

\section{Viral detection in cardiac sarcoidosis - implications for resolving the enigma}

Among the key findings in the present study is the detection of replicating viruses within a lesion of cardiac sarcoidosis (Fig. 3), which has the potential to redefine our understanding of the underlying etiology of this enigmatic, multi-organ disease entity. Here, we demonstrated the presence of HEV and EBV RNA in a lesion of clinically diagnosed cardiac sarcoidosis, in addition to the detection of EBV, CMV and AAV2 in a patient with spontaneous cardiac sarcoidosis in the context of transplant rejection. To our knowledge, this is the first report of actively replicating virus within a granulomatous lesion consistent with sarcoidosis. Such findings could implicate viruses as antigenic triggers for the underlying granulomatous inflammatory response, the hallmark of sarcoidosis. Currently, immunosuppressive therapy is standard of care treatment for sarcoidosis, despite inconclusive evidence of its benefit $^{64,65}$. Thus, as with many modalities of heart failure, therapy is largely supportive, involving standard of care heart failure medications, mechanical assist devices and cardiac transplantation $^{66,67}$. Our findings speak to the need for further investigation in order to rule out viral etiology as a pathogenic trigger responsible for causing genetically susceptible individuals to develop cardiac and systemic sarcoidosis.

\section{Parvovirus B19 - bystander or contributor?}

In the current study, B19V was detected in 3.8\% (3/78) of tissues (including healthy controls) and in $4.3 \%(3 / 69)$ of patients with heart failure. Our study revealed the presence of actively replicating B19V among $12.5 \%(2 / 16)$ of patients with DCM and $20 \%(1 / 5)$ of patients with eosinophilic myocarditis, while it was not detected in patients with a clinical diagnosis of lymphocytic myocarditis (viral or non-viral). The prevalence of B19V among patients with DCM or myocarditis in our cohort is considerably lower than the prevalence quoted in previous reports ${ }^{18,47}$. A recent meta-analysis demonstrated that the prevalence of $B 19 \mathrm{~V}$ in endomyocardial biopsies of patients with myocarditis or DCM 

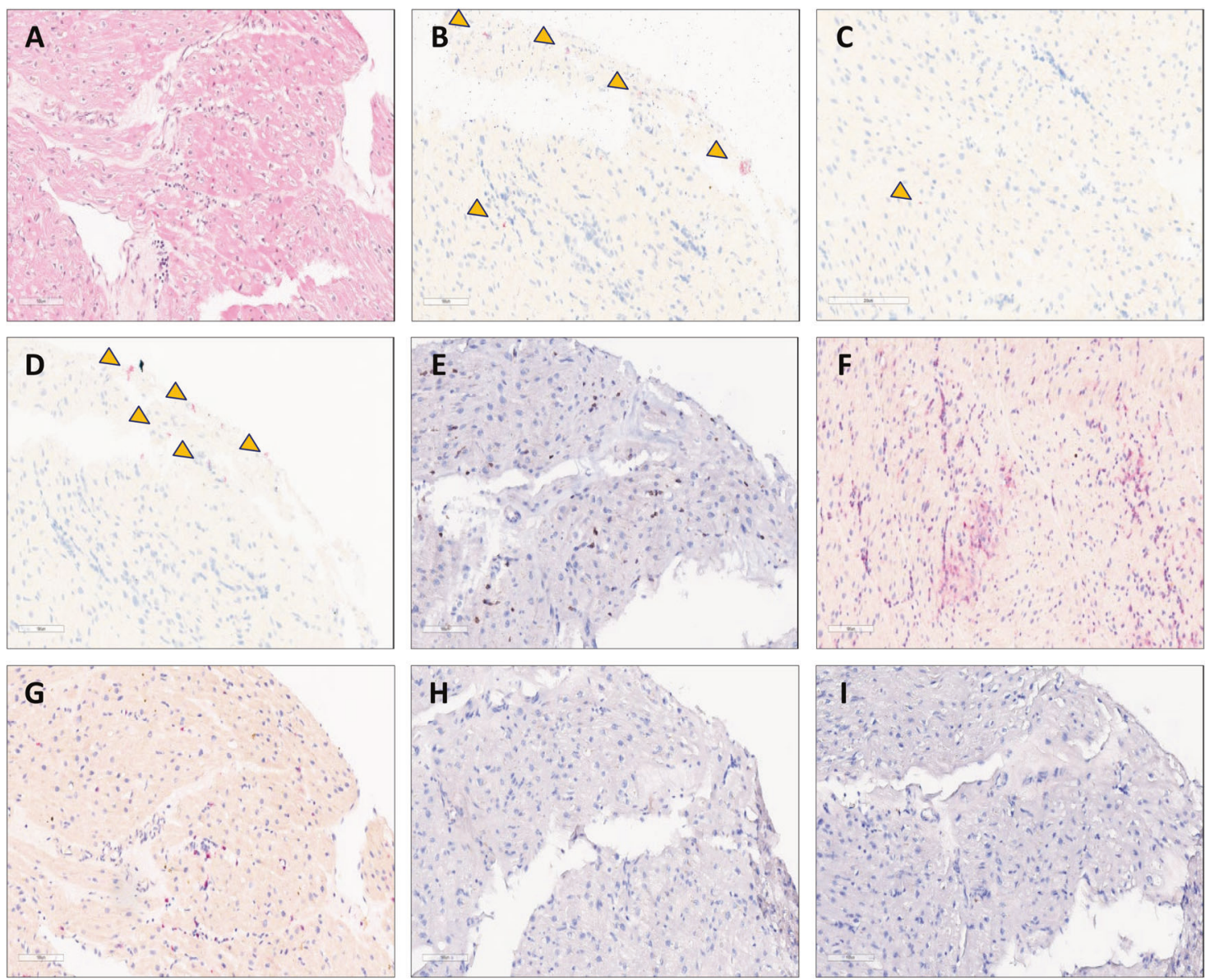

Fig. 5 Adeno-associated virus 2 (AAV2) RNA detected in transplant reject heart with spontaneous cardiac sarcoidosis. A Hematoxylin and Eosin staining of tissue from a patient with a transplant-rejected heart with sarcoidosis. Detection of AAV2 (B), CMV (C) and EBV (D) RNA (red signals) via RNAscope indicated by arrows. IHC staining of regions with detectable viral RNA for CD3 (E), CD4 (F), CD8 (G), CD45 (H) and CD68 (I) as markers of T cells co-receptor, Helper T cells, Natural Killer (NK) cells, leukocyte common antigen and macrophages respectively. Positive signals are indicated in brown $(\mathbf{E}, \mathbf{H}, \mathbf{I})$ and red $(\mathbf{F}, \mathbf{G})$. Representative images $(\mathbf{E}-\mathbf{I})$ were captured from regions with detectable AAV2 and EBV RNA at a 20x magnification.

(45.5\%) was not significantly different than its prevalence in control tissues (37.5\%), which supports the notion of B19V being a bystander virus as opposed to a causative agent for such conditions ${ }^{47,68}$. It is noteworthy that of all three patients in our cohort positive for B19V (2 DCM and 1 eosinophilic myocarditis), at least one additional actively replicating virus was detected within the same tissue. Our study thus supports that B19V acts as a bystander virus, or at least that actively replicating B19V is at a much lower prevalence than has been reported in previous studies, which were reliant upon PCR for detection and did not provide spatial or morphological context of actively replicating viruses within the heart.

\section{An advanced method of cardiotropic virus detection}

The contribution of cardiotropic viruses in initiation and exacerbation of heart failure is often not suspected or confirmed clinically and therefore is likely significantly underreported. Moreover, when virus-associated heart failure is suspected clinically (most commonly in myocarditis), current diagnostic techniques such as PCR are insufficient to detect viral RNA in the heart in a manner that would affirm causation. As such, PCR does not provide the spatial context ${ }^{36,69}$ (i.e., viral localization in the tissue) needed to affirm causation for perceived virus-induced damage. In addition, PCR has considerable potential for false positivity ${ }^{46,70}$, as viral presence in the blood is indistinguishable from viral presence in tissue ${ }^{71}$, making it exceedingly difficult to implicate viruses as potential pathogenetic agents. For example, EBV is among the most prevalent, ubiquitous infections worldwide $^{48}$. Thus, it is difficult to determine whether EBV infection is causative under certain conditions of heart failure or whether the virus is merely a bystander. Although in rare cases EBV has been implicated in acute myocarditis ${ }^{49}$, exacerbation of coronary events ${ }^{50}$ and heart failure ${ }^{51}$, definitive evidence of causation remains elusive. Further, these studies were reliant upon detection of neutralizing antibodies for EBV in patient blood. The method of in situ hybridization demonstrated here corrects for and addresses these challenges by providing morphological context as to whether virus is present in the tissue and in which cell types, while simultaneously distinguishing between replicating and template strands of viral RNA and detecting low copy RNA. Our method thus applies a highly sensitive technique of in situ hybridization capable of detecting single copies of RNA with high specificity and reduced background. While our study has found that viral prevalence in patients with end-stage heart failure is much more common than currently reported, causation cannot be attributed. Accordingly, our research may serve to inspire future research in order to determine the causative relationship between viruses and various heart failure conditions.

In the present study, we designed and developed advanced methodology for the detection of cardiotropic viruses in a heart 
failure cohort to examine the prevalence and phenotype of cardiotropic viruses associated with a spectrum of conditions. Specifically, we designed and developed a custom tissue microarray from explanted and autopsy hearts with a variety of clinically diagnosed, pathologist-confirmed, acute inflammatory (viral myocarditis, lymphocytic myocarditis, eosinophilic myocarditis, transplant rejection, sarcoidosis, GCM), chronic (chronic active myocarditis in the context of DCM, non-inflammatory DCM and (AD) and genetic (HCM, ARVC) conditions from selected regions of pathological significance. Subsequently, we employed a newly emerging in situ hybridization technology uniquely capable of detecting a single RNA molecule with high specificity to identify a panel of common cardiotropic viruses. This method further provides spatial context within our cohort of heart failure patients, creating a snapshot of viral prevalence among various heart conditions. Overall, AAV2 was the most prevalent virus in our cohort, detected in $27.5 \%(19 / 69)$ of patients with heart failure. AAV2 was associated with concurrent infection in $68.4 \%(13 / 19)$ of virus-positive tissues and was detected in $44.4 \%$ (4/9) of viral myocarditis, $66.7 \%(4 / 6)$ of "non-viral" lymphocytic myocarditis, $16.7 \%(1 / 6)$ of chronic active myocarditis (in the context of DCM), $31.3 \%(5 / 16)$ of non-inflammatory DCM, 25.0\% (1/4) of ARVC, $33.3 \%(2 / 6)$ of CAD, 25\% (1/4) of HCM, and $14.3 \%(1 / 7)$ of transplant rejection cases. AAV2 and CMV were both detected in one patient described above, which likely contributed to the exacerbation of orthotropic rejection. The finding of AAV2 in the context of transplant rejection is consistent with previous literature ${ }^{52,61,72}$. We additionally report several novel findings, including the first account of the detection of HCV and EBV within a lesion of cardiac sarcoidosis, and the presence of CVB3 in GCM and the overall presence of viruses across a spectrum of heart failure manifestations, all of which warrant further investigation. Considering the high rate of virus detection in our cohort $(46.4 \%$ among heart failure patients), the presence of virus within the heart is currently associated with poor prognosis. Thus, the combination of a TMA and a highly specific and sensitive in situ hybridization method yields a powerful, high-throughput diagnostic tool which may be implemented in clinical settings to identify pathogenetic viral agents in the context of heart failure. In turn, implementing such as standard of care in the settings of patients for whom reoccurring endomyocardial biopsy is performed may provide avenues for personalized treatment (i.e., antivirals versus immunosuppressive agents) and better our understanding of the role of viruses as initial triggers and in the exacerbation of heart failure conditions previously disregarded to have a viral component in their pathogeneses and progressions.

Limitations. Our cohort of heart failure patients is diverse, which is representative the variability of diagnosis among patients at our research center and in the general population. Patient selection also resulted from the availability of samples in our biobank. As a result of the limited sample size and high heterogenity among patient clinical presentation, even among those with the same diagnoses, some of our research results have only been observed in a small cohort of patients. For example, our novel finding of viral involvement in cardiac sarcoidosis was only reported in one patient due to these limitations. As such, our research is a starting point for further investigation of the role of cardiotropic viruses in heart failure, for which it is recommended that larger sample sizes of more homogenous patient tissues are utilized for pathological review.

Another limitation of the current study was not including an exhaustive panel of all known cardiotropic viruses, including human herpesvirus 6 (HHV6), another virus responsible for causing cardiomyopathy. However, our rather extensive panel of cardiotropic viruses includes those that have historically and regionally been shown to play a role as initial triggers and in the exacerbation of heart failure.

\section{DATA AVAILABILITY}

The datasets used and/or analyzed during the current study are available from the corresponding author on reasonable request.

\section{REFERENCES}

1. Virani, S. S. et al. Heart disease and stroke statistics-2020 update: a report from the American Heart Association. Circulation 141, e139-e596 (2020).

2. Hamm, N. C. et al. Original quantitative research Trends in chronic disease incidence rates from the Canadian Chronic Disease Surveillance System. Health Promot. Chronic Dis. Prev. Can. 39, 216 (2019).

3. Lv, S. et al. Epidemiology and diagnosis of viral myocarditis. Hellenic J. Cardiol. 54, 382-391 (2013).

4. Friman, G. \& Fohlman, J. The epidemiology of viral heart disease. Scand. J. Infect. Dis. Suppl. 88, 7 (1993).

5. Gibbons, C. L. et al. Measuring underreporting and under-ascertainment in infectious disease datasets: a comparison of methods. BMC Public Health 14, 147 (2014).

6. Pischke, S. et al. Hepatitis E in Germany-an under-reported infectious disease. Dtsch. Arztebl. 111, 577 (2014).

7. Fung, G., Luo, H., Qiu, Y. \& Yang, D. McManus B. Myocarditis. Circ. Res. 118, 496-514 (2016).

8. Chow, L. H., Radio, S. J., Sears, T. D. \& Mcmanus, B. M. Insensitivity of right ventricular endomyocardial biopsy in the diagnosis of myocarditis. J. Am. Coll. Cardiol. 14, 915-920 (1989).

9. Schultz, J. C., Hilliard, A. A., Cooper, L. T. Jr \& Rihal, C. S. Diagnosis and treatment of viral myocarditis. Mayo Clin. Proc. 84, 1001-1009 (2009).

10. Ukimura, A., Ooi, Y., Kanzaki, Y., Inomata, T. \& Izumi, T. A national survey on myocarditis associated with influenza H1N1pdm2009 in the pandemic and postpandemic season in Japan. J. Infect. Chemother. 19, 426-431 (2013).

11. Alhogbani, T. Acute myocarditis associated with novel Middle east respiratory syndrome coronavirus. Ann. Saudi. Med. 36, 78-80 (2016).

12. Xiong, T. Y., Redwood, S., Prendergast, B. \& Chen, M. Coronaviruses and the cardiovascular system: acute and long-term implications. Eur. Heart J. 41 1798-1800 (2020).

13. Yamauchi-Takihara, K. What we learned from pandemic H1N1 influenza A. Cardiovasc. Res. 89, 483-484 (2010)

14. Tao, Z. et al. Coxsackievirus B3, Shandong Province, China, 1990-2010. Emerg. Infect. Dis. 18, 1865-1867 (2012).

15. Yang, Y. et al. The deadly coronaviruses: the 2003 SARS pandemic and the 2020 novel coronavirus epidemic in China. J. Autoimmun. 109, 102434 (2020).

16. Palacios, G. \& Oberste, M. S. Enteroviruses as agents of emerging infectious diseases. J. Neurovirol. 11, 424-433 (2005).

17. Bowles, N. E. et al. Detection of viruses in myocardial tissues by polymerase chain reaction. evidence of adenovirus as a common cause of myocarditis in children and adults. J. Am. Coll. Cardiol. 42, 466-472 (2003).

18. Verdonschot, J. et al. Relevance of cardiac parvovirus B19 in myocarditis and dilated cardiomyopathy: review of the literature. Eur. J. Heart Fail. 18, 1430-1441 (2016).

19. Matsumori, A., Shimada, T., Chapman, N. M., Tracy, S. M. \& Mason, J. W. Myocarditis and heart failure associated with hepatitis $C$ virus infection. J. Card. Fail. 12, 293-298 (2006)

20. Premkumar, M. et al. Acute viral hepatitis e is associated with the development of myocarditis. Case Rep. Hepatol. 2015, 458056 (2015).

21. Ace, O. \& Domb, S. Myocarditis as the initial presentation of Epstein-Barr virus infection in a 17-year-old male patient. Can. Fam. Phys. 65, 897-899 (2019).

22. Dickey, T., Schweir, M. \& Hysell, M. Fatal Influenza B Myocarditis in a $34-$ Year-Old Female. Clin. Pract. Cases Emerg. Med. 2, 219-222 (2018).

23. Yoshimizu, N. et al. Repetitive fulminant influenza myocarditis requiring the use of circulatory assist devices. Intern. Med. 53, 109-114 (2014).

24. Ukimura, A., Satomi, H., Ooi, Y. \& Kanzaki, Y. Myocarditis associated with influenza A H1N1pdm2009. Influenza Res. Treat. 2012, 351979 (2012).

25. Kuhl, U. et al. High prevalence of viral genomes and multiple viral infections in the myocardium of adults with "idiopathic" left ventricular dysfunction. Circulation 111, 887-893 (2005).

26. Calabrese, F., Basso, C., Carturan, E., Valente, M. \& Thiene, G. Arrhythmogenic right ventricular cardiomyopathy/dysplasia: is there a role for viruses? Cardiovasc. Pathol. 15, 11-17 (2006).

27. Bowles, N. E., Ni, J., Marcus, F. \& Towbin, J. A. The detection of cardiotropic viruses in the myocardium of patients with arrhythmogenic right ventricular dysplasia/ cardiomyopathy. J. Am. Coll. Cardiol. 39, 892-895 (2002).

28. Lee, K. K. et al. Global burden of atherosclerotic cardiovascular disease in people with hepatitis $C$ virus infection: a systematic review, meta-analysis, and modelling study. Lancet Gastroenterol. Hepatol. 4, 794-804 (2019). 
29. Babiker, A. et al. Risk of cardiovascular disease due to chronic hepatitis $C$ infection: a review. J. Clin. Transl. Hepatol. 5, 343-362 (2017).

30. Panhwar, M. S. et al. Relation of concomitant heart failure to outcomes in patients hospitalized with influenza. Am. J. Cardiol. 123, 1478-1480 (2019).

31. Kytomaa, S. et al. Association of influenza-like illness activity with hospitalizations for heart failure: the atherosclerosis risk in communities study. JAMA Cardiol. 4, 363-369 (2019).

32. Topol, E. J. COVID-19 can affect the heart. Science 370, 408-409 (2020).

33. Inciardi, R. M. et al. Cardiac involvement in a patient with coronavirus disease 2019 (COVID-19). JAMA Cardiol. 5, 819-824 (2020).

34. Chen, $\mathrm{T}$. et al. Clinical characteristics of 113 deceased patients with coronavirus disease 2019: retrospective study. BMJ 368, m1091 (2020).

35. Madjid, M., Safavi-Naeini, P., Solomon, S. D. \& Vardeny, O. Potential effects of coronaviruses on the cardiovascular system: a review. JAMA Cardiol. 5, 831-840 (2020).

36. Sekhri, V., Sanal, S., Delorenzo, L. J., Aronow, W. S. \& Maguire, G. P. Cardiac sarcoidosis: a comprehensive review. Arch. Med. Sci. 7, 546-554 (2011).

37. Fussner, L. A. et al. Management and outcomes of cardiac sarcoidosis: a 20-year experience in two tertiary care centres. Eur. J. Heart Fail. 20, 1713-1720 (2018).

38. Heidecker, B. et al. Virome sequencing in patients with myocarditis. Circ. Heart Fail. 13, e007103 (2020).

39. Duréault, A. et al. Severe infections in sarcoidosis: incidence, predictors and longterm outcome in a cohort of 585 patients. Medicine 96, e8846 (2017).

40. Hepatitis, C. Virus-associated extrahepatic manifestations in lung and heart and antiviral therapy-related cardiopulmonary toxicity. Viral. Immunol. 30, 633-641 (2017).

41. Teragaki, $M$. et al. Prevalence of hepatitis $C$ virus infection among patients with hypertrophic cardiomyopathy. Heart Vessels 18, 167-170 (2003).

42. Shauer, A. et al. Acute viral myocarditis: current concepts in diagnosis and treatment. ISR Med. Assoc. J. 15, 180-185 (2013).

43. Elliott, P. Diagnosis and management of dilated cardiomyopathy. Heart 84, 106(2000).

44. Schultz, J. C., Hilliard, A. A., Cooper, L. T. Jr. \& Rihal, C. S. Diagnosis and treatment of viral myocarditis. Mayo Clin. Proc. 84, 1001-1009 (2009).

45. Murphy, J. \& Bustin, S. A. Reliability of real-time reverse-transcription PCR in clinical diagnostics: gold standard or substandard? Expert. Rev. Mol. Diagn. 9, 187-197 (2009).

46. Seidman, M. \& McManus, B. Myocarditis. Cardiovasc. Pathol. 4, 341-359 (2016).

47. Rigopoulos, A. G. et al. Systematic review of PCR proof of parvovirus B19 genomes in endomyocardial biopsies of patients presenting with myocarditis or dilated cardiomyopathy. Viruses 11, 566 (2019).

48. Kuri, A. et al. Epidemiology of Epstein-Barr virus infection and infectious mononucleosis in the United Kingdom. BMC Public Health 20, 912 (2020).

49. Canter, C. E. \& Simpson, K. E. Diagnosis and treatment of myocarditis in children in the current era. Circulation 129, 115-128 (2014).

50. Binkley, P. F. et al. Evidence for the role of Epstein Barr Virus infections in the pathogenesis of acute coronary events. PLoS One 8, e54008 (2013).

51. Mutlu, H., Alam, M. \& Ozbilgin, O. F. A rare case of Epstein-Barr virus-induced dilated cardiomyopathy. Heart Lung 40, 81-87 (2011).

52. Verdonschot, J. et al. Relevance of cardiac parvovirus B19 in myocarditis and dilated cardiomyopathy: review of the literature. Eur. J. Heart Fail. 18, 1430-1441 (2016).

53. Yajima, T. \& Knowlton, K. U. Viral myocarditis: from the perspective of the virus. Circulation 119, 2615-2624 (2009).

54. Andréoletti, L., Lévêque, N., Boulagnon, C., Brasselet, C. \& Fornes, P. Viral causes of human myocarditis. Arch Cardiovasc. Dis. 102, 559-568 (2009).

55. Esfandiarei, M. \& McManus, B. M. Molecular biology and pathogenesis of viral myocarditis. Annu. Rev. Pathol. Mech. Dis. 3, 127-155 (2008).

56. Pankuweit, S., Lamparter, S., Schoppet, M. \& Maisch, B. Parvovirus B19 genome in endomyocardial biopsy specimen. Circulation 109, e179 (2004).

57. Matsumori, A. Hepatitis C virus infection and cardiomyopathies. Circ. Res. 96, 144-147 (2005).

58. Mahrholdt, $\mathrm{H}$. et al. Cardiovascular magnetic resonance assessment of human myocarditis: a comparison to histology and molecular pathology. Circulation 109, 1250-1258 (2004).

59. Marchant, D. J. \& McManus, B. M. Regulating viral myocarditis: allografted regulatory $T$ cells decrease immune infiltration and viral load. Circulation 121, 2609-2611 (2010).

60. McManus, B. et al. Progress and challenges in the pathological diagnosis of myocarditis. Eur. Heart J. 12, 18-21 (1991).

61. Shirali, G. S. et al. Association of viral genome with graft loss in children after cardiac transplantation. N. Engl. J. Med. 344, 1498-1503 (2001).

62. Breinholt, J. P. et al. Viral epidemiologic shift in inflammatory heart disease: the increasing involvement of parvovirus B19 in the myocardium of pediatric cardiac transplant patients. J. Heart Lung Transplant. 29, 739-746 (2010).
63. Colvin, M. M. et al. Antibody-mediated rejection in cardiac transplantation: emerging knowledge in diagnosis and management. Circulation 131, 1608-1639 (2015).

64. Sadek, M. M., Yung, D., Birnie, D. H., Beanlands, R. S. \& Nery, P. B. Corticosteroid therapy for cardiac sarcoidosis: a systematic review. Can. J. Cardiol. 29, 1034-1041 (2013).

65. Kandolin, R. et al. Cardiac sarcoidosis: epidemiology, characteristics, and outcome over 25 years in a nationwide study. Circulation 131, 624-632 (2015).

66. Donsky, A. S., Escobar, J., Capehart, J. \& Roberts, W. C. Heart transplantation for undiagnosed cardiac sarcoidosis. Am. J. Cardiol. 89, 1447-1450 (2002).

67. Birnie, D. H., Nery, P. B., Ha, A. C. \& Beanlands, R. S. Cardiac sarcoidosis. J. Am. Coll. Cardiol. 68, 411-421 (2016).

68. Koepsell, S. A., Anderson, D. R. \& Radio, S. J. Parvovirus B19 is a bystander in adult myocarditis. Cardiovasc. Pathol. 21, 476-481 (2012).

69. Lin, F. R. et al. Incidence of and factors associated with false positives in laboratory diagnosis of norovirus infection by amplification of the RNAdependent RNA polymerase gene. PLoS One 9, e109876 (2014).

70. Pankuweit, S. \& Klingel, K. Viral myocarditis: from experimental models to molecular diagnosis in patients. Heart Fail. Rev. 18, 683-702 (2013).

71. Basso, C., Calabrese, F., Angelini, A., Carturan, E. \& Thiene, G. Classification and histological, immunohistochemical, and molecular diagnosis of inflammatory myocardial disease. Heart Fail. Rev. 18, 673-681 (2013).

72. Ivan, D., Frazier, O. H. \& Abrams, J. Fatal disseminated adenoviral infection in an adult heart transplant patient. J. Heart Lung Transplant. 23, 1209-1212 (2004).

\section{ACKNOWLEDGEMENTS}

The authors would like to thank Amrit Samra for her contributions to histological work, as well as Rebecca Chan and Henry $\mathrm{Ng}$. The authors also express gratitude to the University of British Columbia, Providence Health Care, and the Center for Heart Lung Innovation at St. Paul's Hospital.

\section{AUTHOR CONTRIBUTIONS}

P.H., F.L., J.H. and B.M. provided study concept and design; P.H., T.M., A.R., F.L., C.N., H.R., V.C., K.A., J.H. and B.M. contributed to development of methodology and writing, review and revision of the paper; P.H., F.L., T.M., A.H., H.R. and B.M. provided acquisition, analysis and interpretation of data, and statistical analysis; P.H. and B.M. provided technical and material support. All authors read and approved the final paper.

\section{FUNDING INFORMATION}

This study was supported by Providence Health Care Research Institute and the St. Paul's Foundation. Dr. Hanson's salary is supported by the Michael Smith Foundation for Health Research and the Myocarditis Foundation.

\section{COMPETING INTERESTS}

K.A. reports grants from ClHR, Canadian Lung Association, Canadian Pulmonary Fibrosis Foundation, Collaborative Health Research Projects (NSERC partnered) and sponsored collaborative research projects and personal fees from Boehringer Ingelheim, sponsored collaborative research projects with Gilead, Patara, Boehringer Ingelheim, Synairgen, Alkermes, GSK, Pharmaxis, Indalo, Unity, Pieris, Pliant, NeRRe, CSL Behring and Bold outside of the submitted work. All other authors declare no competing interests.

\section{ETHICS APPROVAL}

Ethical protocols (H05-50004, H15-40080) approved by the University of British Columbia's Clinical Research Ethics Board were followed. This study was performed in accordance with the Declaration of Helsinki.

\section{ADDITIONAL INFORMATION}

Correspondence and requests for materials should be addressed to Paul J. Hanson.

Reprints and permission information is available at http://www.nature.com/ reprints

Publisher's note Springer Nature remains neutral with regard to jurisdictional claims in published maps and institutional affiliations. 